\title{
An interesting case of Persistent Mullerian Duct Syndrome- An incidental finding
}

\author{
Natarajan Suresh ${ }^{1}$, HemalathaGanapathy ${ }^{2}$, Shubhranshu Shekhar ${ }^{3}$, Prakashiny ${ }^{4}$ \\ 1.Assistant Professor, Department of Pathology, SreeBalaji Medical College and Hospital, Chennai-44 \\ 2.Professor, Department of Pathology, SreeBalaji Medical College and Hospital, Chennai-44 \\ 3. Assistant Professor, Department of Pathology, SreeBalaji Medical College and Hospital, Chennai-44 \\ 4.Post Graduate,Department of Pathology, SreeBalaji Medical College and Hospital, Chennai-44
}

\begin{abstract}
Persistent Mullerian Duct Syndrome is a rare autosomal recessive disease characterized by persistent Mullerian derivative in patients with $46 X Y$ Karyotype and normal virilization . Here we are presenting a case of incidental finding of Persistent Mullerian Duct Remnants in a 58 yr old man during routine hernioplasty.Histological diagnosis was confirmed by Immunohistochemistry

Keywords: Persistent Mullerian Duct Syndrome(PMDS),Hernia uteri inguinale
\end{abstract}

\section{Introduction}

Persistent Mullerian Duct Syndrome is a rare autosomal recessive disease characterized by persistent Mullerian derivative in patients with $46 \mathrm{XY}$ Karyotype and normal virilization ${ }^{1}$. It was first reported by NILSON in $1939^{2}$.These remnants are usually incidental findings during routine herniorraphy for inguinal hernia. PMDS is a form of male pseudohermaphroditism .It is referred to as hernia uteri inguinale ,when it presents as inguinal hernia ${ }^{1}$

\section{Case Report}

A 58yr old man was admitted with swelling, pain and dragging sensation in right inguinal region for past 2 years. After clinical examination surgeons arrived at a diagnosis of right sided indirect inguinal hernia.He had no sexual dysfunction and was fertile, with two sons.On examination his secondary sex characters were well developed .The patient had normal masculine features , moustache,beard,pubic and axillary hair and penis.The right testis was well developed with right sided indirect inguinal hernia.The left scrotum was normal A standard hernioplasty was done and the hernial sac contents were sent for histopathological examination

\section{Pathological Findings}

Gross examination revealed a tubular structure measuring $5.5 \mathrm{~cm}$ in length.The external surface was congested.Cut surface revealed a lumen(Table/Fig1)

Microscopic examination showed tubular structures lined by cuboidal to columnar epithelium resembling endometrial glands surrounded by muscular stroma. Fragments of fibrovascular stroma were also seen.(Table/Fig2)

A provisional diagnosis of Rudimentary Persistent Mullerian Duct structures was made

An IHC panel of CD10 and ER were done. This showed columnar cells showing nuclear positivity for ER(Table/Fig4) and stromal cells positivity for CD10(Table/Fig3)

This confirmed the presence of Rudimentary Persistent Mullerian Duct structures

\section{Discussion}

Persistent Mullerian Duct Syndrome or hernia uteri inguinale term originally used by NILSON in 1939 characteristically described a group of patients with 46XY karyotype and normal male external genitalia but internal mullerian structures . Typically these phenotypes were unilateral or bilateral undescended testis or bilateral fallopian tubes,uterus or upper vagina draining into prostatic utricle ${ }^{2}$

Regression of mullerian duct derivatives usually occurs between 8-10 weeks under the influence of Anti Mullerian Hormone(AMH), a member of TGF beta family and secreted by Sertoli cells.AMH signalling acts through AMH receptor type 2. AMH mutations or loss of AMH Receptor type 2 expression can result in mullerian duct derivatives being inappropriately retained in males ${ }^{3}$

AMH gene is located on ch19p13.3 and AMH Receptor type 2 has been mapped to ch12q13 ${ }^{4}$ PMDS $^{5}$

A 27 base pair deletion of AMH Receptor type 2 gene has been found to be the most common cause of

Serum AMH assay allows clinical discriminations between AMH mutations but is not informative after puberty ${ }^{6}$ hence was not effective in our case 
3 anatomic variants have been described .In the most common variant, one testis is usually found in the scrotum, and the uterus and ipsilateral fallopian tubes in the inguinal canal .In some cases contralateral testis and tube are also in the hernia sac called transverse testicular ectopia ${ }^{7}$. The least common type is characterized by bilateral cryptorchidism with testis embedded in broad ligaments in an ovarian position with respect to the uterus, which is fixed in the pelvis

The treatment of PMDS requires correct diagnosis of the condition and distinguishing it from other intersex disorders . In all such cases ultrasonography,CTscan,MRI are helpful in addition to karyotyping, and testicular biopsies .This helps to ascertain the genetic sex and presence of functional testicular tissue.Serum AMH levels also help in diagnosis before puberty ${ }^{9}$

After confirmation of the diagnosis ,treatment consists of removal of the mullerian duct remnants with orchiopexy or orchiodectomy, as cases of malignancies in mullerian duct remnants have been reported ${ }^{8}$.

\section{Conclusion}

Surgeons dealing with inguinal hernia should be alert to the possibility of PMDS in the event of coming across unexpected findings especially in elderly patients as in our case.A proper followup and thorough investigations to determine Ectopic Mullerian Duct remnants should be conducted

This was an interesting case as patients with mullerian remnants are usually infertile but in this case the patient was fertile with 2 children.Such cases are rare.One such case was reported by MehrdadMohammadiSichani et $\mathrm{al}^{9}$

This case is being presented so that proper recognition and identification of the condition by chromosomal studies,imaging ,karyotyping and hormonal assay is done timely,especially in prepubertal age as fertility can be preserved and future risk of malignancy can be averted

\section{References}

[1]. TuragaKK,StPeterSDCalkins CM,HolcombGW3 rd,OstlieDJ,SnyderCL.Hernia uterus inguinale;a proposed algorithm using the laparoscopic approach.SurgLaparoscEndoscPercutan Tech 2006 p 3826-7

[2]. Diamond Andrew David,Yu N Richard; Sexual Differentiation ,Normal and Abnormal CAMPBELL-WALSH Urology $10^{\text {th }}$ Ed $\operatorname{pg} 3624$

[3]. Larsen,Schoenwolf ; Larsen Human Embryology Ed 2009 pg 509

[4]. BelvilleC,JaminSP,Picard JY : Role of Type 1 receptor for Anti Mullerian hormone in the SMAT 1 sertoli cell line. ONCOGENE 24 pg 4984-4992, ed 2005

[5]. ImbeaudS,BelvilleC,Messika-Zeitoun,HUM MOL Genet 1996 pg 1269-1277

[6]. Belville C, Van Vlifmen H : Mutations of AMH gene in patients with PMDS ,Biosynthesis,secretion,and processing of the abnormal proteins and analysis using a 3D model. MOL Endocrinol 18 pg708-721 ed 2004

[7]. Thompson ST,Grillis MA,Wolkoff LH : TTE in a man with PMDS Arch Pathol Lab Med 118 pg 752-755 ed 1994

[8]. Romero FR,Fucs M ,Castro MG ,Garcia CR ,FernandesRde C ,Perez MD Adenocarcinoma of persistent mullerian duct remnants,case report and differential diagnosis Urology 2005;66;194-5

[9]. MehrdadMohammadiSichani,MitraHeidarpour,AsgharDadkhah,MehranRezvani:PersistentMullerian Duct Syndrome With an irreducible Inguinal Hernia Urol J.2009 298-300

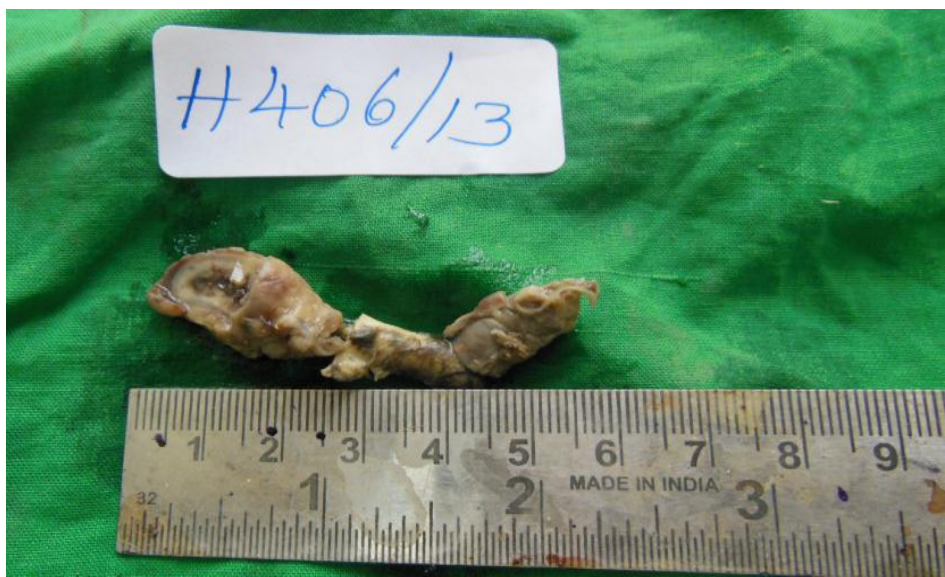

Table/fig 1- a tubular structure measuring $5.5 \mathrm{~cm}$ in length.The external surface was congested. 


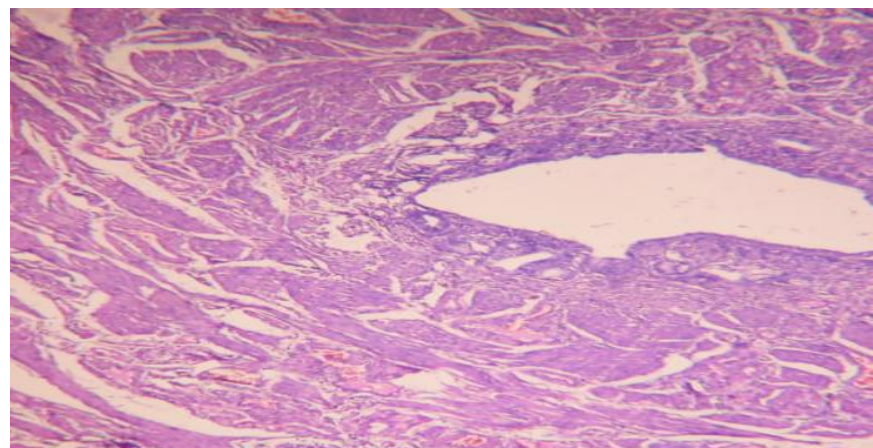

.(Table/Fig2) tubular structures lined by cuboidal to columnar epithelium resembling endometrial glands surrounded by muscular stroma. Fragments of fibrovascularstroma were also seen (H\&E 100X)
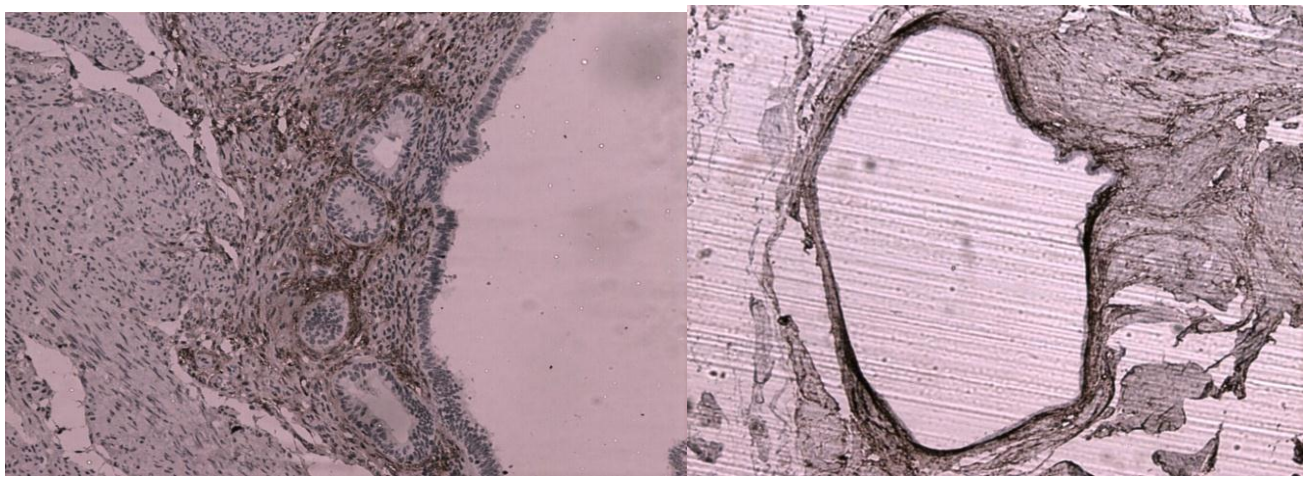

Table/Fig3A,3B: IHC stain shows stromal cell positivity for CD10 (40X view)
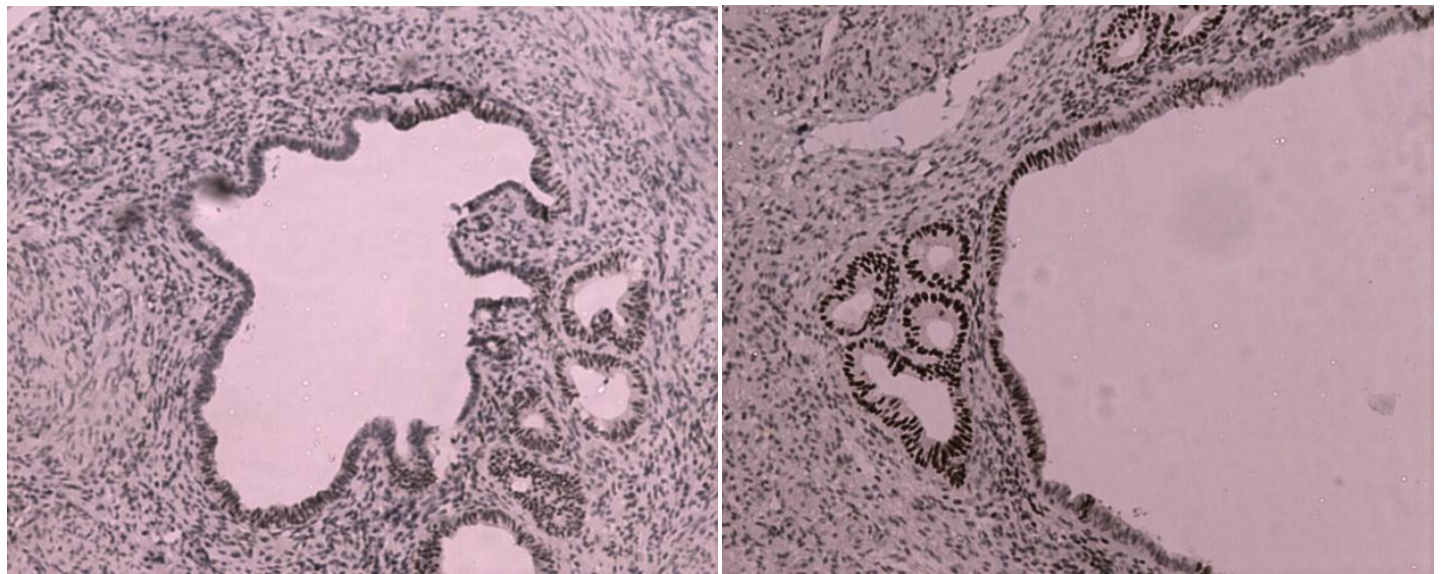

Table/Fig 4A, 4B: Showing nuclear positivity for ER(10X\&40X VIEW) 